\title{
Using Simulation Modeling to Inform Strategies to Reduce Breast Cancer Mortality in Black Women in the District of Columbia
}

\author{
Aimee M. Near, ${ }^{1}$ Jeanne S. Mandelblatt, ${ }^{1}$ Clyde B. Schechter, ${ }^{2}$ and Michael A. Stoto ${ }^{3}$ \\ ${ }^{1}$ Department of Oncology, Georgetown University Medical Center and Cancer Control Program, Lombardi Comprehensive Cancer \\ Center, 3300 Whitehaven Street NW, Suite 4100, Washington, DC 20007, USA \\ ${ }^{2}$ Departments of Family and Social Medicine and Epidemiology and Population Health, Albert Einstein College of Medicine, \\ 1300 Morris Park Avenue, Mazer Building 406, Bronx, NY 10461, USA \\ ${ }^{3}$ Department of Health Systems Administration, Georgetown University, 3700 Reservoir Road, NW Room 236, Washington, \\ DC 20057-1107, USA
}

Correspondence should be addressed to Aimee M. Near, amn45@georgetown.edu

Received 30 October 2011; Accepted 26 April 2012

Academic Editor: Carolyn Rutter

Copyright (C) 2012 Aimee M. Near et al. This is an open access article distributed under the Creative Commons Attribution License, which permits unrestricted use, distribution, and reproduction in any medium, provided the original work is properly cited.

\begin{abstract}
Background. Black women in the District of Columbia (DC) have the highest breast cancer mortality in the US. Local cancer control planners are interested in how to most efficiently reduce this mortality. Methods. An established simulation model was adapted to reflect the experiences of Black women in DC and estimate the past and future impact of changes in use of screening and adjuvant treatment. Results. The model estimates that the observed reduction in mortality that occurred from 1975 to 2007 attributable to screening, treatment, and both was $20.2 \%, 25.7 \%$, and $41.0 \%$ respectively. The results suggest that, by 2020 , breast cancer mortality among Black women in DC could be reduced by $6 \%$ more by initiating screening at age 40 versus age 50 . Screening annually may also reduce mortality to a greater extent than biennially, albeit with a marked increase in false positive screening rates. Conclusion. This study demonstrates how modeling can provide data to assist local planners as they consider different cancer control policies based on their individual populations.
\end{abstract}

\section{Introduction}

Achieving the Healthy People 2020 goals of decreasing breast cancer mortality by $10 \%$ and reducing cancer disparities [1] will require concerted action at the local level, where resources are allocated and programs implemented. The District of Columbia (DC) has the highest female breast cancer mortality rate in the United States, and Black women with breast cancer in the District die at rates that are $43 \%$ higher than Whites with this disease [2]. Since Blacks constitute almost two-thirds of the population in DC, this disparity represents a very large number of excess deaths.

The causes of this disparity are not readily apparent and are not totally explained by differences in known biological factors, incidence, or the use of mammography [3-5]. For instance, in 2007, the incidence rate for DC White woman was 30\% higher than for DC Black women [5], and the available data suggests that Black women in DC are screened at rates comparable to those of White women in the District [4]. In the absence of evidence on the optimal path for eliminating the observed disparities, local cancer control planners requested that an established population simulation model $[6,7]$ be adapted to DC-specific data to identify and evaluate the impact of strategies for reducing breast cancer mortality in Black DC women. The results are intended to inform efforts to decrease breast cancer mortality in the District and to illustrate how modeling can be used to inform and assist local decision makers in evaluating different strategies.

\section{Materials and Methods}

SPECTRUM (Simulating Population Effects of Cancer Control inTerventions-Race and Understanding Mortality) is a simulation model developed within the National Cancer 
Institute's (NCI) Cancer Intervention and Surveillance Modeling Networks (CISNET). The model has been described in detail elsewhere $[6,7]$, and information about the model can also be found online [8].

Briefly, the SPECTRUM model is a discrete event continuous-time population model. This means that women from different birth cohorts are simulated one at a time, and the times at which relevant events occur are determined by sampling from prespecified time-interval distributions. Women who are destined to develop breast cancer may either be screen detected, present with clinical symptoms, or die of other causes before breast cancer is diagnosed. For women who do get breast cancer, the cancer has a stage assigned at presentation, based on whether the tumor is screen or clinically detected. Any effect of screening on survival is the result of stage shift (and, to a lesser extent, age shift in presentation). Ductal carcinoma in situ (DCIS) is represented as a state that can regress, remain and be diagnosed, or progress to invasive cancer [6].

At diagnosis, cancers are designated as being estrogenreceptor (ER), and human epidermal growth factor receptor2 (HER-2) positive or negative. Survival with breast cancer is conditional on age, race, and American Joint Committee on Cancer (AJCC) stage at diagnosis, ER status, HER-2 status, and treatment effectiveness. The unobservable parameters of the model were calibrated to observed incidence and stage distributions in SEER when the model was developed, to capture unmodeled background mortality trends by comparing the observed and model-predicted mortality [7]. Recently, the SPECTRUM model was adapted to project race-specific results [6]. For purposes of this analysis we use the Black model since White women in DC exhibit breast cancer mortality rates comparable to the national White rates, while Blacks in DC have higher death rates than the national average [2]. As a result, local planners were interested in targeting interventions to the Black population. The SPECTRUM model was modified to local data by replacing or adapting relevant parameters to represent Black women in Washington, DC.

2.1. DC-Specific Inputs. The age distribution of the Black female population in DC was based on data from the American Community Survey Public Use Microdata Sample (PUMS). This data is used to initialize the model and generate the correct age distribution of women at the beginning of the simulation [9].

The incidence of breast cancer in the absence of screening was estimated with the use of an age-period-cohort (APC) model as described elsewhere [10]. The APC model was adapted for Black women using an age-specific relative risk of Black versus White incidence [6]. This method has been validated in our prior research [6]. Since the DC tumor registry does not include sufficient years of incidence data to calculate age-, period-, and cohort-specific rates, we compared the trend line of the cross-sectional DC Black breast cancer incidence rates estimated by the DC registry to that of the US incidence rates over time from 1996 to 2007 (Figure 1). Then we calculated a ratio of the DC incidence

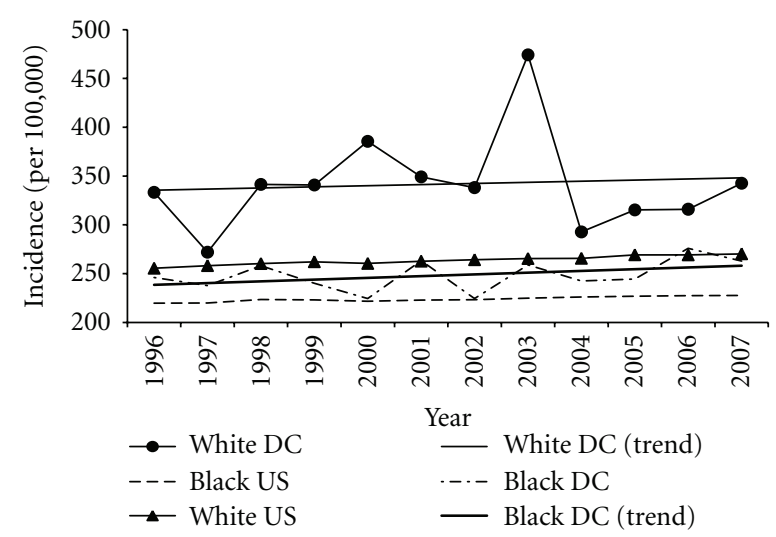

Figure 1: Age-adjusted breast cancer incidence among Black and White women as observed in DC and the US, ages 25 and older, 1996-2007.

(from the trend line) versus US incidence over time since observed data showed that the ratio of DC to US Black breast cancer incidence was constant over time. The average incidence ratio (1.109) was then applied to the Black APC incidence rates model from the SPECTRUM national racespecific analysis to generate the incidence model for the DC Black population. These methods generate a higher incidence for Blacks in DC versus Blacks overall in the US consistent with the observed trends.

We used data on other cause mortality rates in DC Black women from 1999 to $2006[11,12]$ to capture competing mortality.

2.2. Non-DC-Specific Inputs. Surveillance, epidemiology and end results (SEER) data for breast cancer-specific survival from 1975 to 1979 were used to model the natural history of breast cancer in the absence of screening and treatment since these data are not available for the District for this time period [6]. To model the development of breast cancer in the absence of screening, we used SEER data for age- and racespecific stage distribution from 1975 to 1979 with a phasein of Breast Cancer Surveillance Consortium (BCSC) [13] data on the stage distribution of clinically detected tumors in recent years (1996-2007) by linear interpolation between 1990 and 2000.

Information on stage at diagnosis during more recent years with routine mammography screening was provided by the DC Cancer Registry [5]. The mode of detection variable in these data was incomplete, so we compared the stage distribution for all diagnosed DC Black women to the national stage distribution for Black women in the BCSC from 1996 to 2007 (Figure 2). Because the distributions were similar, we modeled the stage distribution of screen-detected women in DC based on the national stage distribution for screen-detected women.

The dissemination of mammography in the US population was estimated in two stages as described in detail elsewhere $[6,14,15]$. Applying this two-part method, the SPECTRUM model first samples an age at first mammography from a nonparametric birth-cohort-specific survival 


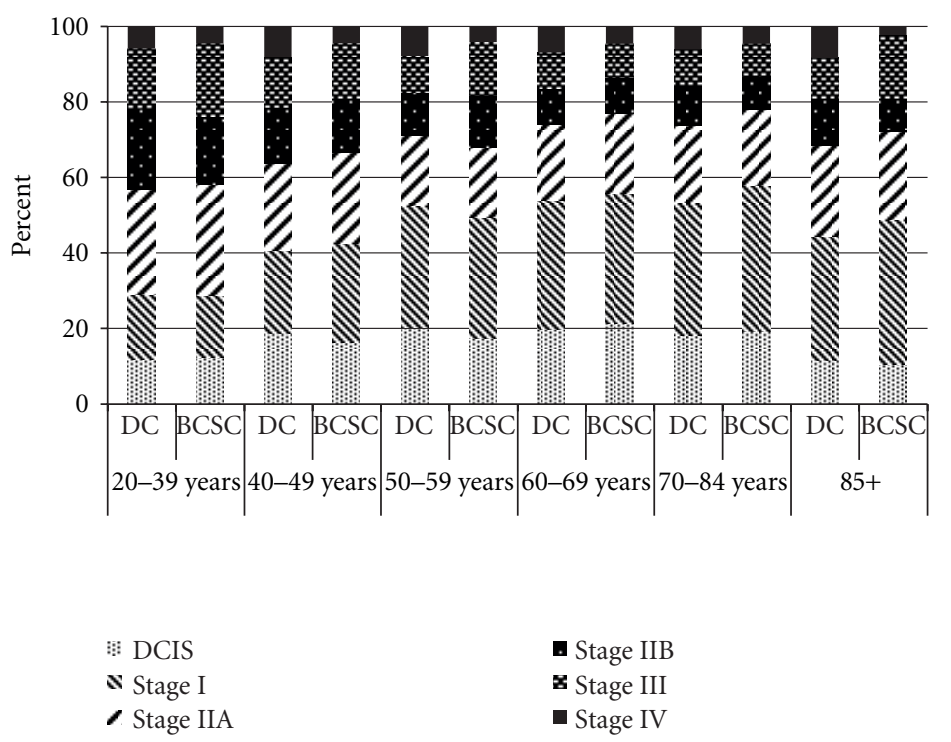

FIGURE 2: Stage distribution of breast cancer cases at diagnosis, Black women, DC and BCSC, 1996-2007, all modes of detection.

function. The second part samples an intermammogram interval from another survival function - this one being dependent on birth cohort and age and varying over the course of a woman's life.

Based on DC-specific Behavioral Risk Factor Surveillance System (BRFSS) data from 1996 to 2008 [4], there does not appear to be a Black-White difference in mammography dissemination in DC (Figure 3). If anything, the BRFSS data suggests that both Black and White women in DC have a higher rate of mammography within the past two years than all US women [4]. Because screening for Black women in DC is comparable to national rates, the national mammography dissemination input was not modified for this analysis. The sensitivity (and specificity) of mammography by 10 -year age group and screening round (initial and subsequent) is based on values observed in the BCSC from 1996 to more recent years. Based on the literature suggesting no effect of race on screening mammography sensitivity and specificity $[16,17]$, we assumed the two measures were equal for Black and White women. Moreover, our past analyses have shown no impact of sensitivity on model results [18]. Finally, in other work we have demonstrated that screening only accounts for $7-8 \%$ of Black-White differences in breast cancer mortality [6].

Data on DC-specific adjuvant therapy use is not collected by the local tumor registry, so we used national data on treatment dissemination. The dissemination of adjuvant therapy by age-, year-, race-, AJCC stage-, ER-, and HER2-status was estimated from data from the NCI's Pattern of Care (POC) studies $[19,20]$ and updated with data from patients receiving treatment at National Comprehensive Cancer Network (NCCN) sites. Data from the POC studies showed Black-White differences in the receipt of multiagent chemotherapy and hormonal therapy, and these differences were applied in the model. Black women were $22 \%$ and $15 \%(10 \%$ for women $<50$ years of age) less likely to receive multiagent chemotherapy and hormonal therapy, respectively [6].
Once treatment is received, there is no evidence that it is any more or less effective in DC than nationally. Thus we used efficacy estimates based on meta-analyses from the Early Breast Cancer Trialists' Collaborative Group [21-23]. Treatment efficacy was assumed to be equal for Blacks and Whites [24].

2.3. Analysis. We conducted four separate analyses. First, we validated the model estimates against observed DC mortality data and our recent analysis of US Black-White differentials [6]. Second, we conducted analyses to partition the impact of screening and treatment on observed breast cancer mortality from 1975 to 2007. To do this, we calculated percent mortality reductions by comparing the mortality in hypothetical scenarios in 2007 with screening, adjuvant treatment, and both with the background mortality predicted by the model in the absence of screening and adjuvant treatment.

Next, motivated by the DC Cancer Control Plan's then current goal of reducing breast cancer mortality rates in the District by $10 \%$, especially among Black women [25], we asked a series of hypothetical questions from local planners about the potential impact of future improvements in screening and treatment. We were interested in whether realistic improvements in cancer control interventions could decrease mortality in Black women by $10 \%$. We modeled 9 different screening and treatment strategies to reflect possible future improvements and compared them to what would occur if current dissemination of screening and treatment remained unchanged. To do so, we modeled dissemination screening and treatment from 1975 to 2010 and then the new screening/treatment scenario from 2011 onward. Improvements in treatment are defined as all women (100\%) receiving the most effective, available treatment, or optimal treatment. Improvements in screening are defined as all women (100\%) being regularly screened, with varying initiation ages (40 versus 50) applied annually or biennially. 


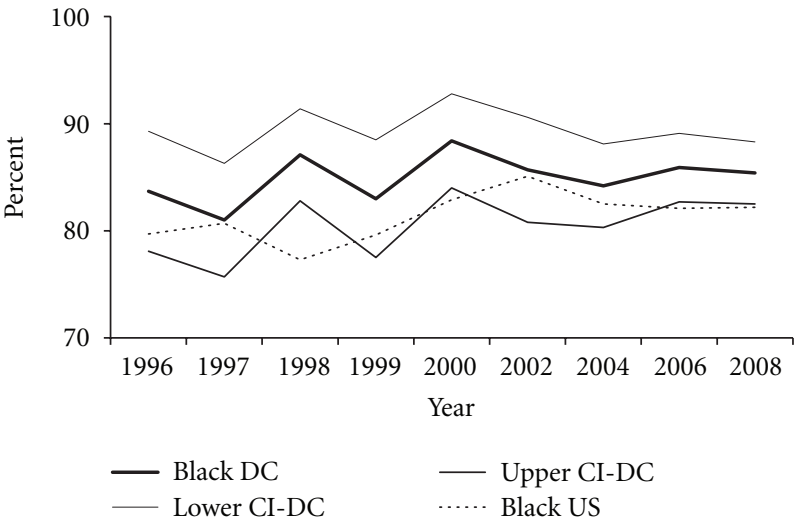

(a)

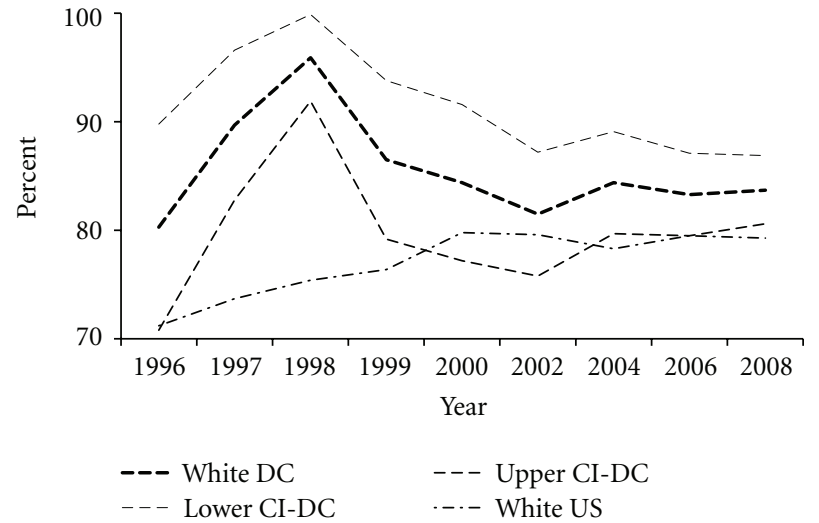

(b)

FIgURe 3: Proportion of Black (a) and White (b) women ages 50 and older who have had a mammogram in the past two years, DC versus US, 1996-2008. DC estimates are displayed with confidence intervals (CIs) [4].

TABLE 1: Contribution of screening and treatment to observed breast cancer mortality reductions* in DC Black women, 2007.

\begin{tabular}{lcc}
\hline $\begin{array}{l}\text { Mortality rate (per } \\
100,000)\end{array}$ & $\begin{array}{c}\text { Percent } \\
\text { reduction from } \\
\text { background } \\
\text { mortality }\end{array}$ \\
\hline No screening or treatment & 76.8 & - \\
Screening only & 61.4 & $20.2 \%$ \\
Treatment only & 57.1 & $25.7 \%$ \\
Screening and treatment & 45.4 & $41.0 \%$ \\
\hline
\end{tabular}

*Mortality reductions (\%) are calculated by comparing the predicted mortality to the background mortality in the scenario without screening and adjuvant therapy.

We compared model results for each of the strategies to select the most efficient approach and compare the benefits and harms of each approach. Screening benefit is represented as the percent mortality reduction (versus current screening dissemination), while harms of regular screening are portrayed in terms of false-positive mammograms.

To rank the strategies, we looked at number of mammograms (the measure of resource use) and the percent mortality reduction. A strategy that requires more mammograms but has a lower relative percent mortality reduction is considered inefficient or dominated by other strategies. As described in prior modeling work [18], we evaluated the strategies and plotted the nondominated strategies on a graph of the number of mammograms versus the percent mortality reduction. The sequence of points representing the largest incremental gain in percent mortality reduction per additional screening is considered the efficiency frontier, with strategies falling on this line being the most efficient.

Finally, to address concerns by planners that the available data may not reflect true disparities in access to health care in the District, we performed a secondary analysis testing the assumption that a substantial proportion of women (50\%) were not screened at all and did not receive optimal adjuvant therapy.
2.4. Outcome Measures. To estimate the impact of future improvements in screening and treatment on breast cancer mortality in DC Black women, we looked at several key outcomes from each model run. The outcomes were presented in terms of the number of mammograms performed, the number of invasive and noninvasive (DCIS) breast cancers that occur, the number of breast cancer deaths averted (all per 100,000 women), the percent reduction in breast cancer mortality, and the number of false positives per 100,000 women and number of mammograms per death averted.

\section{Results}

3.1. Model Validation. Breast cancer incidence among Black women in DC remained relatively stable from 1996 to 2007, and this trend was accurately reproduced by the model (Figure 4). The observed age-adjusted breast cancer mortality rates for Black women in DC diverge only slightly from the rates predicted by the model using actual screening and treatment rates, with the observed mortality being minimally higher than the predicted rates (Figure 5). Lastly, the model predicted a similar stage distribution as was observed for Black women in DC.

3.2. Relative Impact of Screening and Adjuvant Therapy on Breast Cancer Mortality. The model predicted that both mammography screening (20.2\% of the observed reduction) and adjuvant treatment (25.7\% of the observed reduction) contributed substantially to the observed reduction in breast cancer mortality among Black DC women, with treatment having a slightly greater impact (Table 1 ). The combination of screening and treatment is estimated to have resulted in a $41.0 \%$ mortality reduction by 2007 compared to what would have occurred if no screening or adjuvant treatment occurred. It is important to note that improved treatment for later stage disease decreases the impact of screening, and therefore the screening and treatment mortality reductions do not simply add up when measuring the effect of both. 


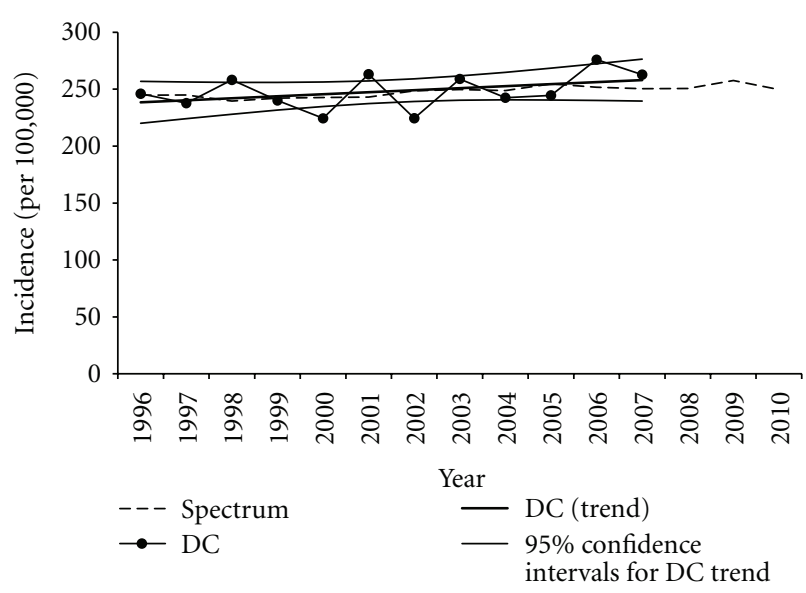

Figure 4: Age-adjusted breast cancer incidence among Black women in DC as observed and predicted by SPECTRUM, ages 25 and older. DC (trend) is based on observed DC breast cancer incidence (DC).

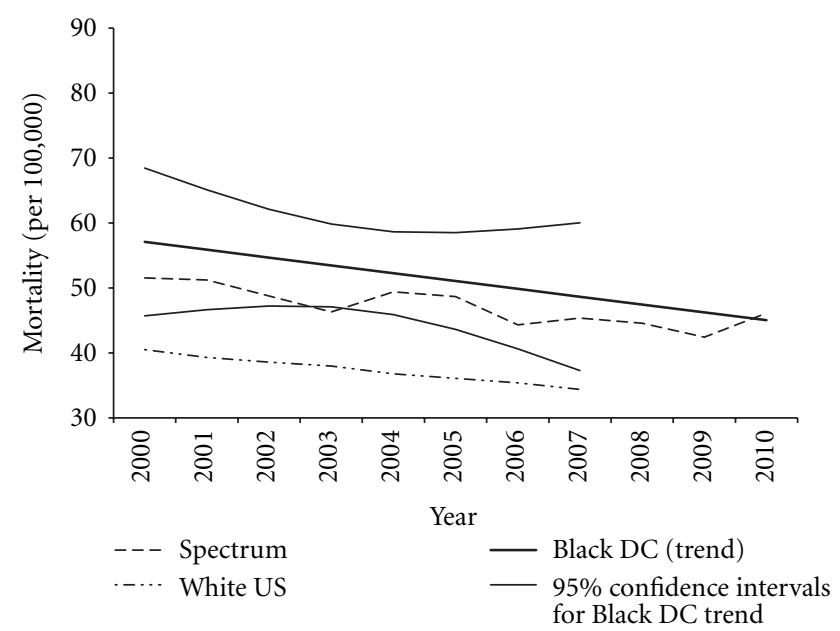

FIGURE 5: Age-adjusted breast cancer mortality for Black women in DC, as observed and predicted by SPECTRUM, ages 25 and older, compared to observed US White mortality. DC (trend) is based on observed DC breast cancer mortality.

3.3. Strategies for Achieving DC Cancer Control Goals. Table 2 summarizes the results of model runs assessing the impact of different potential strategies for reducing breast cancer mortality rates among Black women in DC. All of the calculations represent patterns that could be expected by 2020 assuming changes started in 2011.

The results suggest that focusing on screening improvements alone, defined as higher rates of regular use, earlier starting ages, and/or annual screening will all decrease mortality but increase the number of false positives substantially. For example, ensuring that all women receive annual screening from ages 40 to 79 is predicted to decrease mortality by $13.8 \%$ (versus actual current screening and treatment) but will increase the number of false positives by 4,436 per 100,000 women. On the other hand, improving treatment so that all women receive optimal adjuvant therapy, but making no changes in screening, may have a similar magnitude of impact on breast cancer mortality (mortality reduction of $15.3 \%$ ), without increasing the number of false positives. Combining both approaches is predicted to result in a $28.8 \%$ mortality reduction with almost the same number of false positives as focusing on screening alone. Furthermore, biennial screening appears to achieve almost as much mortality reduction as annual but would produce many fewer false positive mammograms (Table 2). In terms of different starting ages for biennial screening, starting at age 40 versus 50 lowers the breast cancer mortality rate from 40 to 39 per 100,000 women but increases the number of false positives from 2,487 to 3,634 per 100,000 women. With annual screening, starting screening at age 40 rather than 50 is predicted to lower the breast cancer mortality rate from 38 to 35 per 100,000 women but increases the number of false positives by 2,482 per 100,000 women.

To illustrate the tradeoff between the number of mammograms performed (and the nearly proportional number of false positives) and mortality reduction for starting screening at age 40 versus 50, Figure 6 presents the results in terms of "efficiency frontiers." Four strategies were considered efficient because they provide additional mortality reductions for added use of mammography. Assuming either actual or optimal treatment patterns, the following screening scenarios are on the efficiency frontier: biennial 50-79, biennial 40-79, and annual 40-79. Screening annually from ages $50-79$ is not as efficient as the other three strategies for either treatment scenario (Figure 6).

Whether one assumes actual (left panel) or optimal (right panel) treatment patterns, the reduction in mortality (vertical axis) is greatest with annual screening beginning at age 40 , but this scenario calls for many more mammograms to be performed. Comparing the left panel to the right panel, it seems that achieving more than a 15\% mortality reduction is not possible without optimal treatment, whereas with optimal treatment, the achievable mortality reduction may be more than $20 \%$ with relatively little additional screening effort.

3.4. Secondary Analysis. We reestimated outcomes under the hypothetical worst case assumption that, from 1975 to 2010, half of all Black women in DC did not get screened at all and that only half as many women got adjuvant therapy compared to the actual data (results not shown). The numerical results in these two scenarios differ, but the order of the strategies with screening annually ages 40-79 and optimal treatment as the most effective approach is the same as in the main analysis. Because of the assumptions of this secondary analysis up until 2011, the benefits in terms of percent mortality reduction due to improved screening and treatment are greater than the main analysis. For example, screening annually ages 40-79 with optimal treatment beginning in 2011 results in a $28.8 \%$ mortality reduction by 2020 in the main analysis and a $40.0 \%$ mortality reduction in the secondary analysis. Varying our assumptions about the current rate of screening and treatment did not change the ranking of the strategies. 


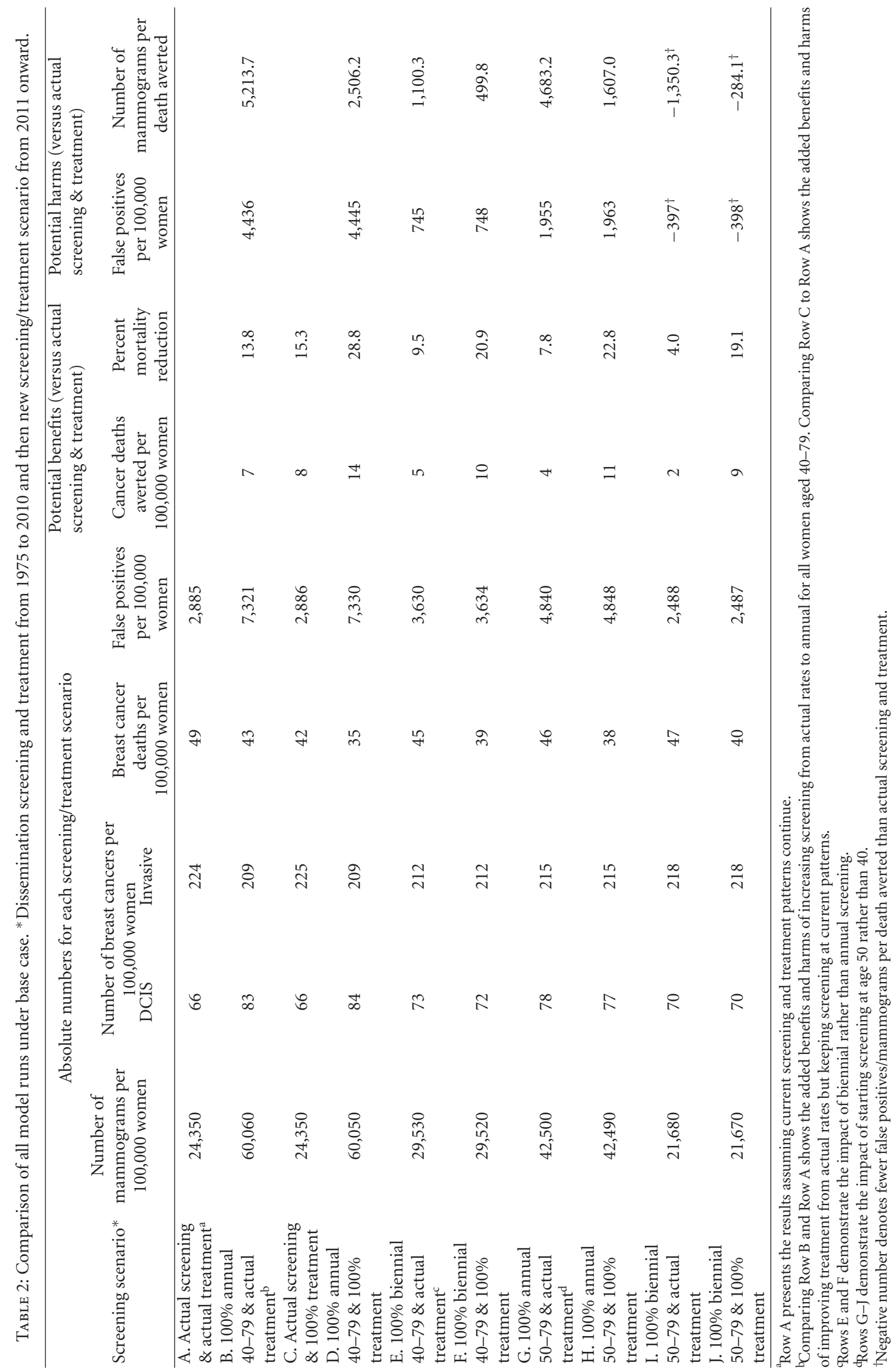




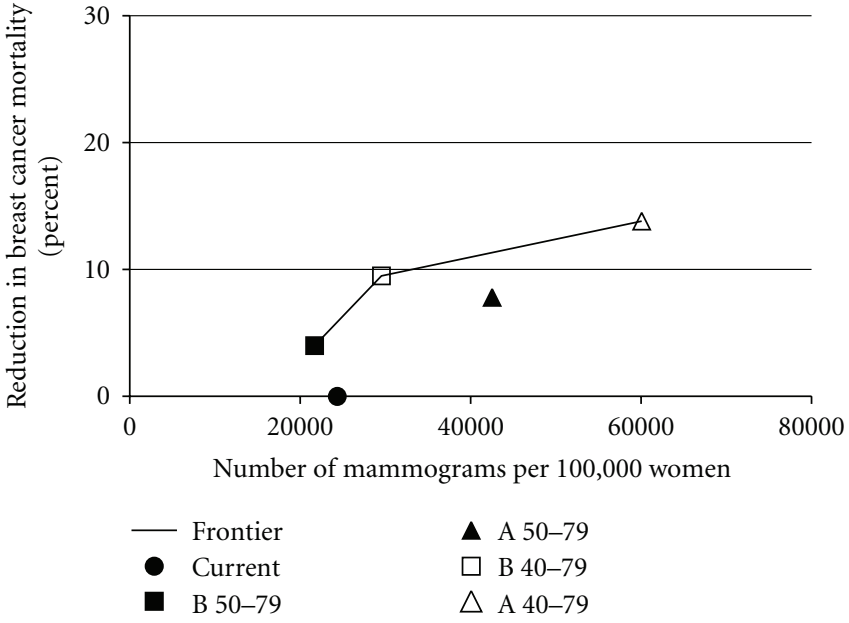

(a)

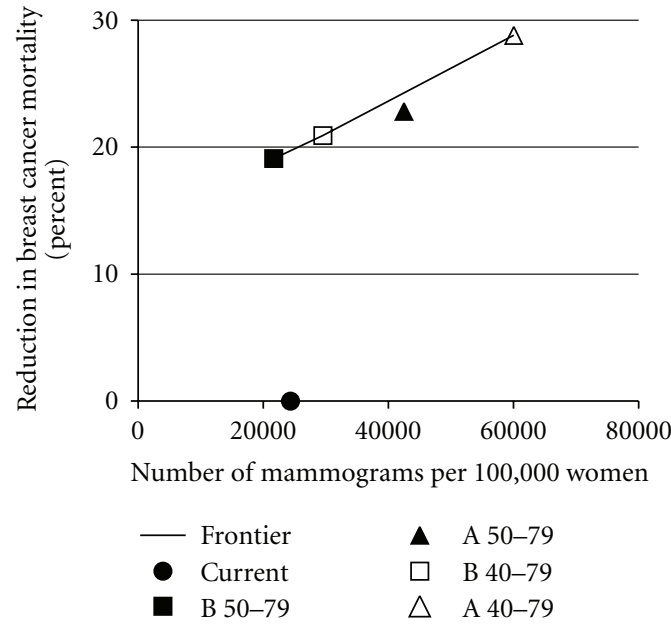

(b)

FIGURE 6: Efficiency frontier comparing screening strategies under 100\% screening compliance among Black women in DC, assuming actual treatment patterns (a) and optimal treatment (b). The line between strategies represents the "efficiency frontier." Strategies on this line would be considered efficient because they achieve the greatest gain per use of mammography compared with the point immediately below it. Points that fall below the line are not considered as efficient as those on the line.

\section{Discussion}

To our knowledge, this is one of the first studies to apply an established national model to simulate cancer control strategies for the elimination of race-based disparities in breast cancer mortality rates in a local area. Our results suggest that in a locality where the minority population is the majority, screening recommendations may vary from those recommended in national guidelines. For instance, we found that breast cancer mortality among Black women in the District of Columbia could be reduced by $6 \%$ more by initiating screening at age 40 versus age 50 . Screening annually may also reduce mortality to a greater extent than biennially, albeit associated with a marked increase in false positive screening rates. However, according to our results, obtaining the greatest mortality reduction will not be achieved through screening alone and must be accompanied by programs that ensure that all women diagnosed receive recommended adjuvant therapy.

Although this is the first time a simulation model has been used to explore racial disparities in a local area, others have utilized simulation models to answer important questions at the city level and in different countries. For example, a breast cancer simulation model known as the "Wisconsin Breast Cancer Epidemiology Simulation Model today" was used to explain breast cancer trends in Wisconsin from 1982 to 1992 [26] and later adapted to national data [27]. In addition, the MISCAN-Fadia (MIcrosimulation of Screening Analysis-Fatal diameter) model was used to assess the cost effectiveness of human papillomavirus vaccination in the Netherlands [28]. This model was also used to estimate the impact of mammography screening in Australia [29], and Florence, Italy [30] and to compare the impact of mammography screening in the Netherlands and the United Kingdom
[31]. However, this is the first time that a model has been used to look at disparities in a local area.

This study suggests that past advances in screening and treatment have accounted for roughly equal amounts of the reduction in breast cancer mortality in Black women in DC-with treatment having perhaps a slightly greater impact. These results are similar, but somewhat higher than those predicted in a national model [6]. The impact of screening has been similar in DC and the US (as expected in light of the similarity in screening inputs), but improved treatment is estimated to have had a somewhat greater effect in DC (25.7\% mortality reduction) than nationally $(21.9 \%$ mortality reduction) [6].

The DC Cancer Control Plan aims to reduce breast cancer mortality in Black DC women by $10 \%$ through strategies targeted to women, health care providers, and the healthcare system intended to increase screening rates, increase access to treatment, and improve treatment outcomes [25]. Our model results suggest that optimal screening and treatment for Black women in DC has the potential to reduce Black breast cancer mortality rates by more than $25 \%$ before 2020 . In fact, 6 (almost 7) of the 9 strategies seem to achieve the originally targeted $10 \%$ mortality reduction by 2020 as set in the DC Cancer Control Plan. Of the two changes, treatment makes a bigger difference, most likely because screening rates are already high in DC Black women. Annual screening starting at age 40 with current treatment is predicted to reduce breast cancer mortality by $13.8 \%$. On the other hand, optimal treatment with current screening is predicted to reduce mortality by $15.3 \%$ without increasing the harm (and costs) of false positives.

This study suggests that obtaining the biggest impacta $28.8 \%$ reduction in mortality-requires both screening and treatment improvements. This involves screening every 
year from age 40 to 79 and providing appropriate adjuvant therapy to every woman diagnosed. However, this strategy will involve far more mammograms and yield more false positives (4,445 per 100,000 women) than the current situation. More false positives occur in screening strategies which involve screening initiation at age 40 rather than 50 and annually versus biennially. For this reason, DC decision makers might want to aim for another efficient approachbiennial screening starting at age 40 -which is suggested to yield almost as much mortality reduction $(20.9 \%)$ with proportionally fewer mammograms and false positives.

While universal optimal treatment may be difficult to achieve, including this as a future goal highlights the fact that without reaching optimal treatment for everyone diagnosed with breast cancer, we may be unable to reduce breast cancer mortality in DC Black women by more than $15 \%$ within the next 8 years. With improved access to and compliance with optimal therapy, a $20 \%$ mortality reduction may be achievable by 2020 with relatively few changes from current screening practices, according to the model.

It is interesting to note that starting screening at age 40 (perhaps biennially) differs from the US Preventive Services Task Force's (USPSTF) 2009 recommendations suggesting that screening not start until age 50 for average risk women $[18,32]$. This result may reflect the fact that Black women in DC have higher than average incidence rates than the US average for Blacks and that Black women in DC have higher mortality rates than other Black women in the US. As the DC Cancer Control Plan suggests, Black women in DC may be diagnosed at a later stage than others because they do not or cannot take advantage of available health care [25]. Black women are more likely to be diagnosed with breast cancer at younger ages than White women [33], and Blacks constitute almost two-thirds of the DC population. Therefore, our data could support a decision for younger screening initiation ages in Black women.

There are a number of limitations to this analysis. First and foremost is the scarcity of DC-specific data. We were unable to find data on adjuvant therapy utilization in the District and therefore assumed that adjuvant therapy rates were the same for DC Black women compared to national rates. We were unable to determine the mode of detection of DC breast cancer cases and generate DC-specific inputs for the stage distribution of screened and clinically detected cases. It is possible that the national data for these parameters is not representative of the DC Black population, which may have resulted in over- or underestimations of the effect of screening and treatment in DC.

Additionally, the only screening data was based on the DC BRFSS [4]. The latter is a telephone survey, and the absence of land lines and other biases may mask a lower screening rate in the overall population of Black women. In addition, the DC Cancer Registry had considerable missing data on variables such as ER/PR and HER-2 status, so we needed to rely on national data for these parameters. To the extent that women in DC have a different disease profile, then screening and treatment could have varying relative effects.

Another limitation of this analysis is the omission of overdiagnosis as an outcome measure. Due to the lack of primary data on the natural history of DCIS and small invasive cancer, we were very conservative in any conclusions about overdiagnosis in prior work [18]. There is also no data to suggest that overdiagnosis is differential by race. To that end, we did not measure the magnitude of overdiagnosis associated with different screening schedules in this study.

Lastly, we did not include the cost of mammograms or treatment in the analysis. Although the number of mammograms (and false positives) serves as a proxy for resource consumption, we did not consider the true economic impact and feasibility of the strategies in the District. Even with these acknowledged limitations, the model demonstrates meaningful outcomes to assist local planners in reducing breast cancer mortality among Black women in DC.

Our results suggest that the optimal strategy for reducing breast cancer mortality rates for Black women in DC involves screening starting at age 40 coupled with a program to ensure that all women found to have breast tumors are optimally treated. Improved screening alone will not enable DC to meet its goals. True benefits will fall short of the model projected results because individual adherence is never perfect. The model does not, however, resolve the question of whether screening should be annual or biennial. The results suggest that the impact of annual screening includes a very substantially greater number of false positives and more than 5 times as many mammograms per death averted and that biennial screening achieves about threequarters of the benefits of annual screening.

\section{Conclusions}

Our results suggest that of the options considered a combination of universal optimal treatment and universal annual screening starting at age 40 will produce the greatest reduction in breast cancer mortality for Black women in DC. Moreover, scaling back the screening to biennial may still reduce mortality enough to achieve twice the DC Cancer Control goal of a $10 \%$ mortality reduction and will do so with far fewer mammograms and false positives. Choices about optimal ages of initiation and cessation will ultimately depend on program goals, resources, the balance of harms and benefits, and considerations of efficiency and equity [18].

\section{Acknowledgments}

The authors thank the National Comprehensive Cancer Network (NCCN) investigators for use of data on treatment dissemination and also the Breast Cancer Surveillance Consortium (BCSC) for the data they have provided for this study. They would also like to thank the following community leaders and local decision makers for providing their expertise and input for this project: Ms. Alisha Baptiste, Dr. John Lynch, Ms. Kathleen Wall, Dr. Donald Henson, Ms. Courtney Clyatt, Ms. Ify Nwabukwu, Ms. Cynthia Bardwell, Ms. Paula Rothenberg and Ms. Katie Hool, Dr. Leslie Calman, Dr. Karen Peterson, Dr. Maimah Karmo, 
Dr. Steven Patierno, Dr. Wayne Frederick, Dr. John DaviesCole, Ms. Alicia Vargas, Ms. Kathleen Rogers, Ms. Amari Pearson-Fields, Dr. LaQuandra Nesbitt, Ms. Beth Beck, Dr. Carole Gresenz, Dr. Michelle Bennett, and Ms. Lenora Johnson. This work was supported by the National Cancer Institute at the National Institutes of Health (Grant no. 2U01CA088283 and U01 CA152958-02.). Breast Cancer Surveillance Consortium data collection was supported by NCI-funded Breast Cancer Surveillance Consortium cooperative agreements (U01CA63740, U01CA86076, U01CA86082, U01CA63736, U01CA70013, U01CA69976, U01CA63731, and U01CA70040).

\section{References}

[1] "Healthy People 2020 (HP2020)," http://www.healthypeople. gov/2020/topicsobjectives2020/objectiveslist.aspx?topicId=5.

[2] SEER $*$ Stat Database: Mortality-All COD. Aggregated With State, Total U.S. (1969-2006), Katrina/Rita Population Adjustment, National Cancer Institute, DCCPS, Surveillance Research Program, Cancer Statistics Branch, 2009.

[3] SEER $*$ Stat Database: Incidence-SEER 9 Regs Research Data. Nov 2008 Sub (1973-2006), Katrina/Rita Population Adjustment, _Linked To County Attributes_-Total US, 1969-2006 Counties, National Cancer Institute, DCCPS, Surveillance Research Program, Cancer Statistics Branch, 2009.

[4] Centers for Disease Control and Prevention (CDC), Behavioral Risk Factor Surveillance System Survey Data, U.S. Department of Health and Human Services, Centers for Disease Control and Prevention, Atlanta, Ga, USA, 1996-2008.

[5] DC Cancer Registry Dataset, District of Columbia Department of Health, Washington, DC, USA, 1996-2007.

[6] N. T. van Ravesteyn, C. B. Schechter, A. M. Near et al., "Racespecific impact of natural history, mammography screening, and adjuvant treatment on breast cancer mortality rates in the United States," Cancer Epidemiology Biomarkers and Prevention, vol. 20, no. 1, pp. 112-122, 2011.

[7] J. Mandelblatt, C. B. Schechter, W. Lawrence, B. Yi, and J. Cullen, "The SPECTRUM population model of the impact of screening and treatment on U.S. breast cancer trends from 1975 to 2000: principles and practice of the model methods," Journal of the National Cancer Institute, no. 36, pp. 47-55, 2006.

[8] http://cisnet.cancer.gov/.

[9] American Community Survey Public Use Microdata Sample (PUMS) 2006-2008 3-Year Downloaded at, http://factfinder. census.gov/home/en/acs_pums_2008_3yr.html.

[10] T. R. Holford, K. A. Cronin, A. B. Mariotto, and E. J. Feuer, "Changing patterns in breast cancer incidence trends," Journal of the National Cancer Institute, no. 36, pp. 19-25, 2006.

[11] "Other cause adjustment references: Centers for Disease Control and Prevention, National Center for Health Statistics," Multiple Cause of Death File 1999-2004. CDC WONDER On-line Database, compiled from Multiple Cause of Death File 1999-2004 Series 20 No. 2J, 2007, http://wonder.cdc. gov/mcd-v2004.html.

[12] "Centers for Disease Control and Prevention, National Center for Health Statistics. Multiple Cause of Death File 2005-2006," CDC WONDER On-line Database, compiled from Multiple Cause of Death File 2005-2006 Series 20 No. 2L, 2009, http://wonder.cdc.gov/mcd-icd10.html.
[13] R. Ballard-Barbash, S. H. Taplin, B. C. Yankaskas et al., "Breast cancer surveillance consortium: a national mammography screening and outcomes database," American Journal of Roentgenology, vol. 169, no. 4, pp. 1001-1008, 1997.

[14] K. A. Cronin, A. B. Mariotto, L. D. Clarke, and E. J. Feuer, "Additional common inputs for analyzing impact of adjuvant therapy and mammography on U.S. mortality," Journal of the National Cancer Institute, no. 36, pp. 26-29, 2006.

[15] K. A. Cronin, B. Yu, M. Krapcho et al., "Modeling the dissemination of mammography in the United States," Cancer Causes and Control, vol. 16, no. 6, pp. 701-712, 2005.

[16] K. S. Gill and B. C. Yankaskas, "Screening mammography performance and cancer detection among black women and white women in community practice," Cancer, vol. 100, no. 1, pp. 139-148, 2004.

[17] D. S. May, N. C. Lee, L. C. Richardson, A. G. Giustozzi, and J. K. Bobo, "Mammography and breast cancer detection by race and Hispanic ethnicity: results from a national program (United States)," Cancer Causes and Control, vol. 11, no. 8, pp. 697-705, 2000.

[18] J. S. Mandelblatt, K. A. Cronin, S. Bailey et al., "Effects of mammography screening under different screening schedules: model estimates of potential benefits and harms," Annals of Internal Medicine, vol. 151, no. 10, pp. 738-747, 2009.

[19] A. Mariotto, E. J. Feuer, L. C. Harlan, L. M. Wun, K. A. Johnson, and J. Abrams, "Trends in use of adjuvant multiagent chemotherapy and tomoxifen for breast cancer in the United States: 1975-1999," Journal of the National Cancer Institute, vol. 94, no. 21, pp. 1626-1634, 2002.

[20] A. B. Mariotto, E. J. Feuer, L. C. Harlan, and J. Abrams, "Dissemination of adjuvant multiagent chemotherapy and tamoxifen for breast cancer in the United States using estrogen receptor information: 1975-1999," Journal of the National Cancer Institute, no. 36, pp. 7-15, 2006.

[21] Early Breast Cancer Trialists' Collaborative Group, "Polychemotherapy for early breast cancer: an overview of the randomised trials," The Lancet, vol. 352, no. 9132, pp. 930942, 1998.

[22] Early Breast Cancer Trialists' Collaborative Group, “Tamoxifen for early breast cancer: an overview of the randomised trials," The Lancet, vol. 351, no. 9114, pp. 1451-1467, 1998.

[23] "Effects of chemotherapy and hormonal therapy for early breast cancer on recurrence and 15-year survival: an overview of the randomised trials," The Lancet, vol. 365, no. 9472, pp. 1687-1717, 2005.

[24] J. J. Dignam, "Efficacy of systemic adjuvant therapy for breast cancer in African-American and Caucasian women," Journal of the National Cancer Institute, no. 30, pp. 36-43, 2001.

[25] DC Cancer Coalition, Facing the Challenge: DC Cancer Control Plan 2005-2010, Department of Health, Washington, DC, USA, 2006.

[26] P. Chang, A Simulation Study of Breast Cancer Epidemiology and Detection since 1982: the Case for Limited Malignant Potential Lesions-Dissertation, University of Wisconsin-Madison, Madison, Wis, USA, 1993.

[27] D. G. Fryback, N. K. Stout, M. A. Rosenberg, A. TrenthamDietz, V. Kuruchittham, and P. L. Remington, "The wisconsin breast cancer epidemiology simulation model," Journal of the National Cancer Institute, no. 36, pp. 37-47, 2006.

[28] I. M. de Kok, M. Van Ballegooijen, and J. D. Habbema, "Costeffectiveness analysis of human papillomavirus vaccination in 
the Netherlands," Journal of the National Cancer Institute, vol. 101, no. 15, pp. 1083-1092, 2009.

[29] R. Carter, P. Glasziou, G. Van Oortmarssen et al., "Costeffectiveness of mammographic screening in Australia," Australian Journal of Public Health, vol. 17, no. 1, pp. 42-50, 1993.

[30] E. Paci, R. Boer, M. Zappa et al., "A model-based prediction of the impact on reduction in mortality by a breast cancer screening programme in the City of Florence, Italy," European Journal of Cancer A, vol. 31, no. 3, pp. 348-353, 1995.

[31] E. van den Akker-van Marle, H. de Koning, R. Boer, and P. Van der Maas, "Reduction in breast cancer mortality due to the introduction of mass screening in the Netherlands: comparison with the United Kingdom," Journal of Medical Screening, vol. 6, no. 1, pp. 30-34, 1999.

[32] "Screening for breast cancer: U.S. Preventive Services Task Force recommendation statement," Annals of Internal Medicine, vol. 151, no. 10, pp. 716-726, 2009.

[33] J. S. Mandelblatt, W. Liang, V. B. Sheppard, J. Wang, and C. Isaacs, "Breast Cancer in minority women," in Diseases of the Breast, J. Harris, M. Lippman, M. Morrow, and C. K. Osborne, Eds., Lippincott Williams \& Wilkins, Philadelphia, Pa, USA, 4th edition, 2009. 


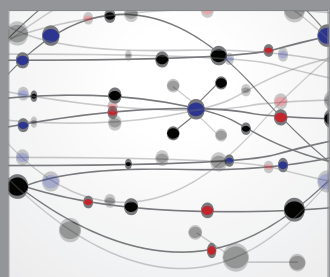

The Scientific World Journal
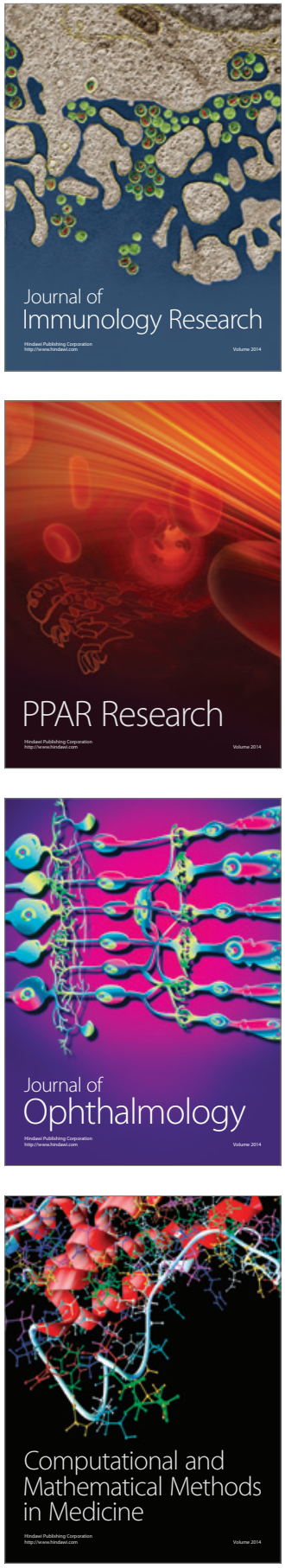

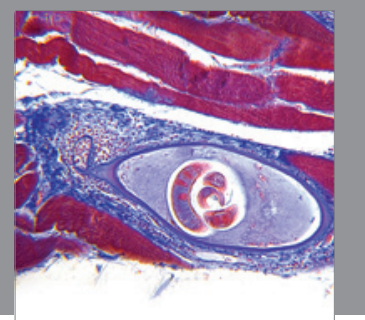

Gastroenterology

Research and Practice
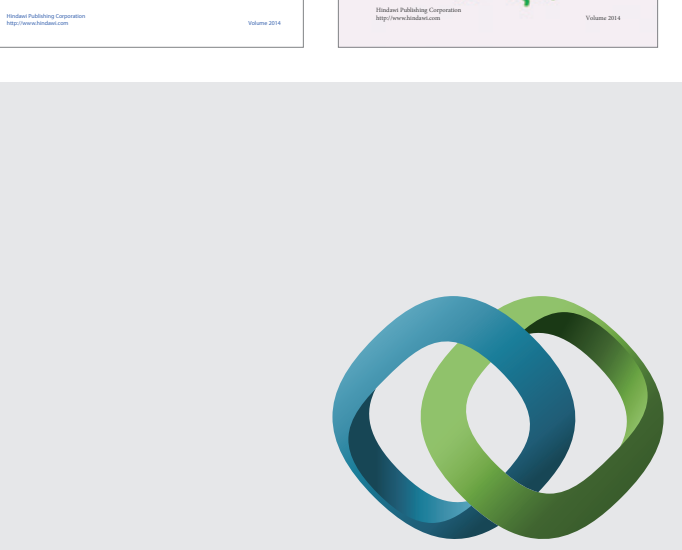

\section{Hindawi}

Submit your manuscripts at

http://www.hindawi.com
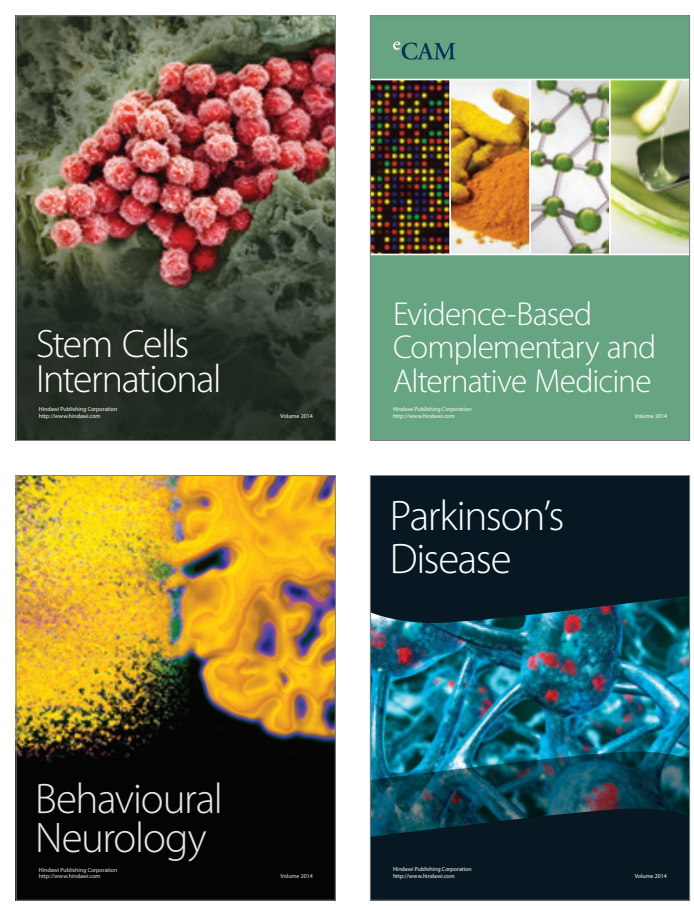

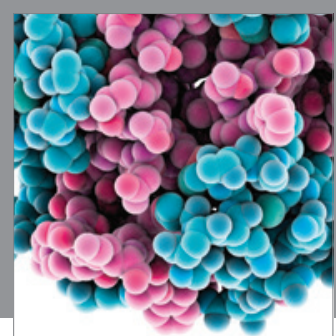

Journal of
Diabetes Research

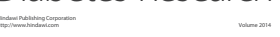

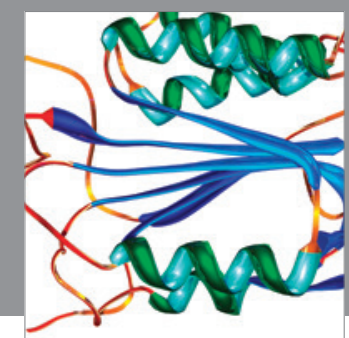

Disease Markers
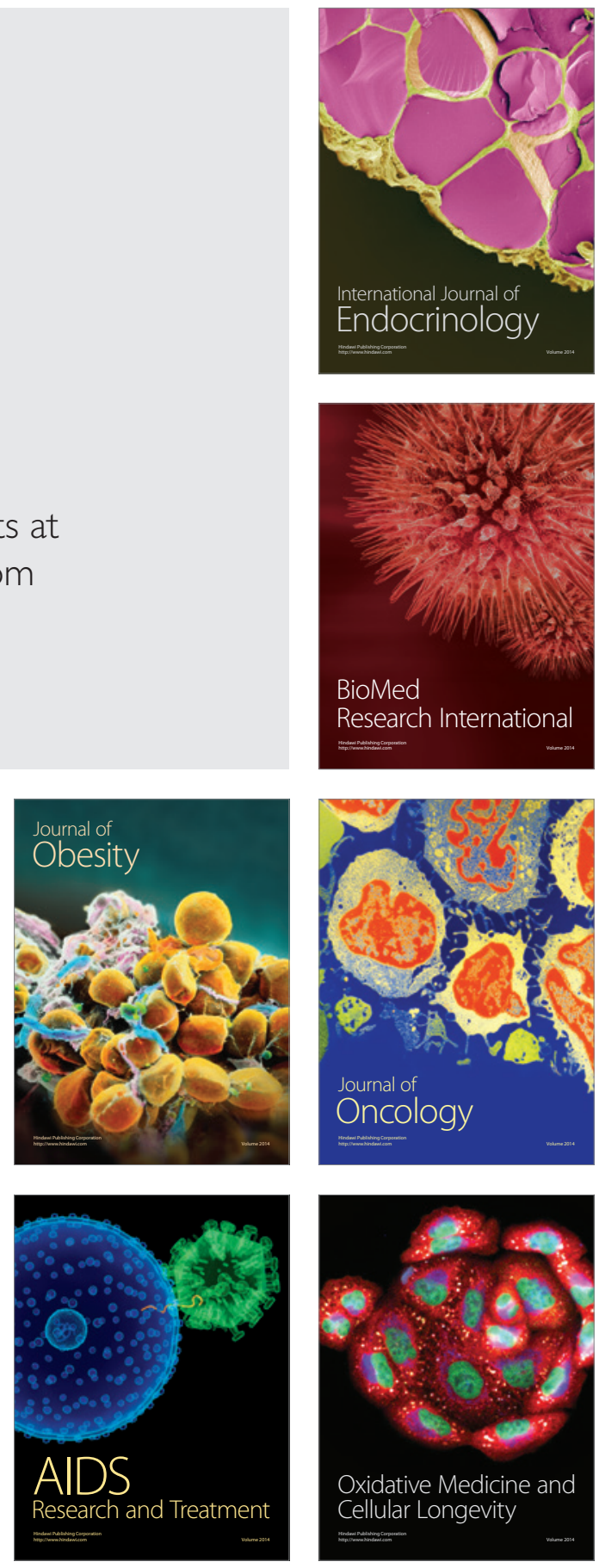